\title{
Direct conversion of human fibroblasts into retinal pigment epithelium-like cells by defined factors
}

\author{
Kejing Zhang ${ }^{1}$, Guang-Hui Liu ${ }^{2 \bowtie}$, Fei $\mathrm{Yi}^{3}$, Nuria Montserrat ${ }^{4,5}$, Tomoaki Hishida ${ }^{1}$, \\ Concepcion Rodriguez Esteban ${ }^{1}$, Juan Carlos Izpisua Belmonte ${ }^{1,4 \bowtie}$
}

${ }^{1}$ Gene Expression Laboratory, Salk Institute for Biological Studies, 10010 North Torrey Pines Road, La Jolla, CA 92037, USA

${ }^{2}$ National Laboratory of Biomacromolecules, Institute of Biophysics, Chinese Academy of Sciences, Beijing 100101, China

${ }^{3}$ Department of Molecular and Cellular Physiology, Stanford University School of Medicine, 265 Campus Drive, Stanford, CA 94305, USA

${ }^{4}$ Center for Regenerative Medicine in Barcelona, Dr. Aiguader 88, 08003 Barcelona, Spain

${ }^{5}$ Biomedical Research Networking Center in Bioengineering, Biomaterials and Nanomedicine (CIBER-BBN), Barcelona, Spain

\ Correspondence: ghliu@ibp.ac.cn (G.-H. Liu), belmonte@salk.edu, izpisua@cmrb.eu (J. C. Izpisua Belmonte)

Received April 20, 2013 Accepted May 21, 2013

\section{ABSTRACT}

The generation of functional retinal pigment epithelium (RPE) is of great therapeutic interest to the field of regenerative medicine and may provide possible cures for retinal degenerative diseases, including age-related macular degeneration (AMD). Although RPE cells can be produced from either embryonic stem cells or induced pluripotent stem cells, direct cell reprogramming driven by lineage-determining transcription factors provides an immediate route to their generation. By monitoring a human RPE specific Best1::GFP reporter, we report the conversion of human fibroblasts into RPE lineage using defined sets of transcription factors. We found that Best1::GFP positive cells formed colonies and exhibited morphological and molecular features of early stage RPE cells. Moreover, they were able to obtain pigmentation upon activation of Retinoic acid (RA) and Sonic Hedgehog (SHH) signaling pathways. Our study not only established an ideal platform to investigate the transcriptional network regulating the RPE cell fate determination, but also provided an alternative strategy to generate functional RPE cells that complement the use

Electronic supplementary material The online version of this article (doi:10.1007/s13238-013-0011-2) contains supplementary material, which is available to authorized users. of pluripotent stem cells for disease modeling, drug screening, and cell therapy of retinal degeneration.

KEYWORDS retinal pigment epithelium, fibroblasts, direct conversion

\section{INTRODUCTION}

The retinal pigment epithelium (RPE) is a pigmented monolayer of epithelium residing outside of the neurosensory retina where it supports metabolic and cellular processes of retinal photoreceptors. Dysfunction and degeneration of RPE lead to photoreceptor loss in many sight-threatening diseases, including the leading causes of blindness in the developed world, age-related macular degeneration (AMD) (Khandhadia et al., 2012). Currently, treatments available for these diseases are limited and do not offer a cure for the cell loss. Therefore, the generation of functional RPE cells is of great therapeutic interest to the field of regenerative medicine and offers possible cures for retina degeneration diseases (Schwartz et al., 2012).

Induced RPE differentiation has been achieved previously using embryonic stem cells (ESCs) and induced pluripotent stem cells (iPSCs) (Carr et al., 2009; Lu et al., 2009; Zhu et al., 2013). Although differentiating RPE cells from hESCs is promising (Zhang et al., 2013), the application of hESCs for therapeutic purposes remains challenging due to un-eased ethical concerns. iPSC technology holds the 
potential to be used to generate patient specific cells for autologous cell transplantation, which has created enormous expectations and circumvented some ethical debates (Takahashi and Yamanaka, 2006; Takahashi et al., 2007). However, differentiation of RPE cells from iPSCs is inefficient, time-consuming, and variable among different iPSC lines (Buchholz et al., 2009). Moreover, iPSCs present several safety concerns such as the genetic and epigenetic aberrations they are carrying, as well as the potential risk for tumor formation (Panopoulos et al., 2011). Recently, advances in direct cell lineage conversion have suggested a potential solution to these issues. The fact that one somatic cell type can be readily converted into another implicates access to abundant resources of any clinically relevant cell type. Moreover, as direct lineage conversion bypasses the pluripotent state, it could theoretically reduce the risk of tumorigenicity after transplantation (Ben-David and Benvenisty, 2011). Using distinct sets of transcription factors, direct lineage conversion technology has been applied to produce various cell types, including neurons, hepatocytes and neural stem cells (Vierbuchen et al., 2010; Huang et al., 2011; Kim et al., 2011; Pang et al., 2011; Sekiya and Suzuki, 2011; Giorgetti et al., 2012; Liu et al., 2012; Zhang et al., 2012).

Here we report the successful development of a RPEspecific Best1::GFP reporter, which faithfully represented human RPE lineage commitment during hESC differentiation. Using this reporter system, we show that a defined set of transcription factors can reprogram human fibroblasts into Best $1:: G P^{+}$colonies. These Best $1:: G^{+} P^{+}$cells exhibit specific morphological and molecular features of RPE lineage and are capable of pigmentation. Our study not only provided a powerful system to study the nature of cellular identity and plasticity of RPE lineage, but also offered a new path to produce functional RPE cells for regenerative therapy and drug development in the future.

\section{RESULTS}

\section{Establishment of a human RPE specific reporter} system

A proper reporter system is essential for monitoring efficient cell fate conversion in reprogramming studies. A series of truncated versions of human Bestrophin1 (Best1) gene and Rpe65 gene promoter, have been shown to mediate robust ocular reporter gene expression that is restricted to the RPE in transgenic mice (Boulanger et al., 2000; Esumi et al., 2004; Acland et al., 2005; Esumi et al., 2009). To test their functionality in human cells, these promoter fragments were cloned into the upstream of EGFP in a lentiviral vector, pGreenZeo (Fig. 1A). We then performed lentivirus infection to test the promoter specificity in different human cell types including human foreskin fibroblasts (HFF), HEK293T cells, H9 hESCs, as well as human primary RPE cells (HRPE). Distinct from the non-specific expression of the Rpe65(0.8k) or Rpe65(1k) reporter in all of the examined cell types, the Best1(0.6 kb) (spanning from -588 to +58$)$ and Best1 $(1 \mathrm{~kb})$ (spanning from -1020 to +38 ) reporters exhibited high selectivity in driving strong EGFP expression in human RPE cells (Fig. 1A). In the following studies, data presented were generated using Best1(0.6 kb) reporter as Best $1:$ GFP reporter, if not specifically indicated. In order to further confirm the specificity of the reporter, we tested the reporter in H9 ESC differentiated cells at day 60 , using a reported protocol (Meyer et al., 2009), which gave rise to a mixed population of various cell types including RPE cells. We observed that the Best1::GFP reporter was only expressed in RPE-like cells, which displayed a morphologically characteristic hexagonal shape, but not in other neural or fibroblast-like cells (Fig. 1B).

We next evaluated whether the Best1::GFP reporter works properly during the directed RPE differentiation from hESCs. Accordingly, H9 hESCs were transduced with Best1::GFP lentiviral reporter and ten individual colonies were chosen to verify the integration of Best1::GFP expression cassette by genomic DNA PCR (data not shown). Positive colonies were subcultured and expanded for further studies. For RPE differentiation, we developed a rapid and efficient protocol, which is modified from two recent publications (Boucherie et al., 2013; Zhu et al., 2013) (Fig. 2A, group Activin A). With this approach, pigmented cells appeared in a monolayer form 3 weeks after Activin A treatment. Immunostaining showed that these cells expressed typical RPE markers including microphthalmia-associated transcription factor (MITF) and Best1, as well as a tight junction protein, ZO-1, indicating a successful commitment of RPE from hESCs (Fig. 2E). Given the reported roles of Retinoic acid (RA) and Sonic Hedgehog (SHH) signaling in RPE maturation and pigmentation (Okada et al., 2004; Zahabi et al., 2012), we set up an RA plus SHH experimental group. As shown in Fig. 2A and 2B, RA plus $\mathrm{SHH}$ treatment enhanced the pigmentation despite resulting in a lower yield of RPE cells compared to the Activin A treatment group.

Monitoring the Best $1:$ GFP expression represents a direct way to visualize the dynamics of RPE differentiation. In both the "Activin A" and "RA plus SHH" treatment groups, Best1:: $\mathrm{GFP}^{+}$cells were initially observed at around day 21 of differentiation, and the percentage of Best $1::$ GFP $^{+}$cells increased to approximate $50 \%$ after 40 days of differentiation (Fig. 2C). Consistently, time-course studies showed that the levels of Best 1 mRNA were strongly upregulated after 3 weeks of differentiation from $\mathrm{H} 9$ hESCs (Fig. 2D). Interestingly, Best1::GFP was highly expressed in non-pigmented and lightly pigmented RPE cells, while when cells were heavily pigmented, GFP fluorescence diminished or even became undetectable (Fig. 2E c-c", d-d"). Since endogenous Best1 protein was still expressed in highly pigmented cells (Fig. 2E d-d"), the absence of GFP fluorescence in these cells may be attributed to the absorption of fluorescence by melanin. To further characterize the mature status 
A

Auman Best1 promoter EGFP
-154 to +38
-294 to +38
-588 to +38
-1020 to +38
Human Rpe65 promoter

B

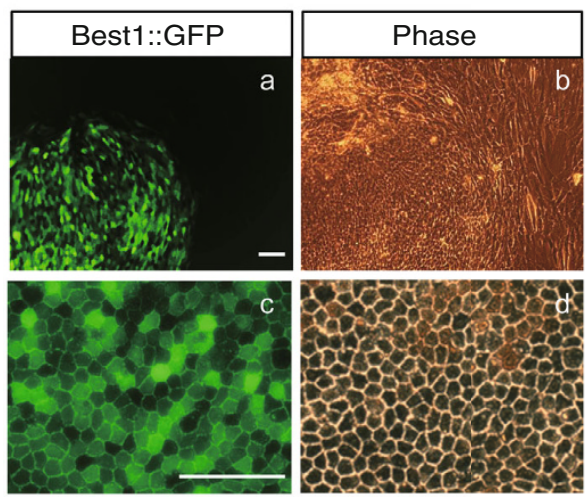

Figure 1. Generating human RPE specific reporters. (A) Schematic representation of the promoter regions of human Best1 or Rpe65 gene for reporter constructs. The table shows the relative GFP levels of each reporter observed in different cell lines. HRPE, human primary RPE cells; -, no expression; +, weak expression; +++, strong expression. (B) Best1::GFP specifically expressed in RPE-like cells differentiated from H9 hESC. All scale bars: $100 \mu \mathrm{m}$.

of Best $1:: \mathrm{GFP}^{+}$cells that followed the protocol with Activin A treatment, we performed GPCR analysis at day 40 . The results demonstrated that Best $1:: \mathrm{GFP}^{+}$cells did express the early eye field genes including Pax6, Lhx2, Six 6 and $R a x$ (Zuber et al., 2003) (Fig. 2F). Two key genes for RPE differentiation, Mitf and Otx2 (Boulanger et al., 2000; Steingrimsson et al., 2004), were markedly upregulated. Other mature RPE hallmark genes were also highly expressed in Best1::GFP ${ }^{+}$cells, including Best1, pigment epithelium derived factor (Pedf), RPE-specific protein $65 \mathrm{kDa}$ (Rpe65), cellular retinaldehyde-binding protein (Cralbp) involved in vitamin A metabolism, tyrosinase (Tyr) and tyrosinase related protein 2 (Tyrp2), both involved in pigment synthesis (Martinez-Morales et al., 2004; Strauss, 2005) (Fig. 2F). These gene expression patterns of Best $1:: \mathrm{GFP}^{+}$cells are similar to those of human primary RPE cells (Fig. 2F). Collectively, these data demonstrate the successful establishment of a human RPE specific reporter system, which faithfully represented RPE lineage commitment during ESC differentiation.

\section{Definition of transcription factor pool}

We hypothesized that transcription factors known to instruct RPE formation during development might also facilitate the conversion of other somatic cell types into RPEs. To test this idea, we constructed retroviral expression vectors encoding six candidate transcription factors (TFs): Rax, Crx, Pax6, Mitf, Otx2, and $\mathrm{Nrl}$ that have been reported to participate in different stages of retina development and RPE specification (Zuber et al., 2003; Martinez-Morales et al., 2004; Steingrimsson et al., 2004) (Fig. 3A). After transduction of HFF with a combination of these 6 TFs together with Best1::EGFP reporter lentivirus, cells were cultured with hESC culture medium (CDF12) to prime the lineage conversion for 7 days. The cells were subsequently cultured in a similar condition that was used to induce RPE differentiation from hESCs (Fig. 3B). However, such a strategy resulted in neither obvious morphological change nor Best $1: \mathrm{GFP}^{+}$cells from parental HFF. This raised a possibility that other factors may be required to increase the epigenetic "plasticity" of the original human fibroblasts. Based on the "clue" that two "Yamanaka factors" (cMyc and Klf4) are highly expressed in adult RPEs (Salero et al., 2012), we supplemented $c M y c$ and $K I f 4$ into the previous six transcription factor cocktail. qPCR analysis verified the expression of all eight transcription factors (8TFs) in HFF transduced with this new cocktail for three days (Fig. 3C). Using the co-transduced retroviral vector encoding mCherry as an indicator, we found that the retrovirus infection system in HFF achieved a transduction efficiency for individual factors of $77 \%$ (Fig. 3D). This experiment suggests that almost $12 \%$ of parental HFF cells could have the opportunity to express all of the eight TFs to initiate the reprogramming process.

\section{Best1::GFP ${ }^{+}$cells generated by direct reprogramming of human fibroblasts}

We next performed the lineage conversion experiments by transduction of HFF with 8TFs. Encouragingly, a number of Best $1: \mathrm{GFP}^{+}$colonies emerged at 12 days post-transduction. These Best1::GFP ${ }^{+}$cell colonies kept actively proliferating and became tightly compacted with a high cytoplasmic to nuclear ratio at day 21. Later, Best $1:: \mathrm{GFP}^{+}$colonies gradually spread out on matrigel and exhibited a cobblestone-like morphology at day 35 (Fig. 4A). These 
morphological observations suggested a RPE progenitorlike status of the generated Best $1:: \mathrm{GFP}^{+}$cell colonies. To determine which of the eight factors were necessarily required for generating Best $1:: \mathrm{GFP}^{+}$colonies, we removed each TF-encoding vector from the 8TFs cocktail one at a time (Fig. 4B). As shown in Fig. 4B, excluding cMyc, MitfA or Otx2 eliminated $\mathrm{GFP}^{+}$colony formation, and the number of Best1::GFP ${ }^{+}$colonies was dramatically reduced when $R a x$, or Crx was removed. Interestingly, when Pax 6 was removed, the number of Best $1::$ GFP $^{+}$colony slightly reduced, while the number of colonies was not discernibly changed when KIf4 or $\mathrm{Nrl}$ was ruled out (Fig. 4B). However, when any combination of two or three factors of Klf4, Pax6 and $\mathrm{Nrl}$ was removed from the $8 T F$ s pool, we could not detect any Best $1:$ : $\mathrm{GFP}^{+}$colonies (data not shown). These data indicated that cMyc, Mitf, Otx2, Rax, and Crx are crucial for reprogramming of human fibroblasts into Best1::GFP ${ }^{+}$cells, while $\mathrm{KIf4}, \mathrm{Nrl}$ and Pax6 individually are not so important and could be omitted or replaced by other factors.

\section{Characterization of HFF-derived Best1::GFP ${ }^{+}$cells}

To assess whether Best $1:: G F^{+}$colonies hold the potential to achieve known characteristics of cultured RPE cells, we evaluated the molecular signatures of the generated Best $1:$ : $\mathrm{GFP}^{+}$cells induced by 8TFs. Immunofluorescence analysis demonstrated that most of the Best $1:: \mathrm{GFP}^{+}$colonies were positive for MITF staining (Fig. 5A). We observed that only some of the colonies expressed Pax6, which is consistent with the notion that down-regulated expression of Pax6 occurs upon RPE maturation (Fig. 5A and data not shown). To induce the further maturation of the Best1::GFP ${ }^{+}$colonies, we picked the Best $1:: \mathrm{GFP}^{+}$colonies onto matrigel coated wells at day 21 , and then the cells were maintained in the medium consisting of Activin A alone, or RA plus $\mathrm{SHH}$ (Fig. 3B). Both conditions promoted further growth of the Best $1:: \mathrm{GFP}^{+}$colonies. At day 35 post-transduction, cells derived from Best1::GFP ${ }^{+}$colonies exhibited cobblestonelike morphology resembling RPE cells differentiated from hESCs, and expressed RPE-specific markers including MITF, ZO-1 and Best1 (Fig. 5A). More importantly, in the RA plus $\mathrm{SHH}$ treatment group, pigmented cells were observed at day 35 , indicating that Best $1:: G F^{+}$cells have achieved further maturation giving rise to functional RPE cells (Fig. 5B). To further characterize their identity, we purified the Best $1:: G^{+} P^{+}$cells by flow cytometry analysis (FACS), and performed qPCR to analyze the transcripts of RPE-specific factors. Compared to the control HFF cells, the generated Best $1:: \mathrm{GFP}^{+}$cells expressed not only the transcription factors we introduced, but also other eye field genes such as Six3 and Lhx2. Additionally, a series of mature RPE marker genes, including Best1, Cralbp, Pedf, and Tyrp2, were also dramatically induced in Best $1:: \mathrm{GFP}^{+}$cells directly converted from HFF, and notably these genes are involved in different functions of RPE, e.g. vitamin A metabolism, pigment synthesis and growth factor secretion etc. (Strauss, 2005) (Fig. 5C). Nevertheless, it should be noted that some of the mature RPE markers such as Rpe65 and Tyr still remained at low expression levels, suggesting the functionally incomplete maturation of these Best $1:: G_{\text {GFP }}{ }^{+}$cells at this stage (Fig. 5C). Taken together, these results indicate that Best1:: $\mathrm{GFP}^{+}$colonies generated from human fibroblasts recapitulated the morphological and molecular features of RPE cells, which, upon further maturation, may represent an invaluable RPE replacement for the field of regenerative medicine.

\section{DISCUSSION}

Encouraged by the first report of the positive implication from prospective clinical trials transplanting hESCs derived RPE cells into two patients (Schwartz et al., 2012), RPE cells generated from pluripotent stem cells have attracted increasing attention for the promise of regenerative medicine (Zhang et al., 2013). Current methods differentiating RPE cells from human pluripotent cells include spontaneous differentiation, monolayer differentiation or 3D culture using mouse and human ESCs (Meyer et al., 2009; Osakada et al., 2009; Nakano et al., 2012). Although several of these recent methods have significantly advanced the yield and accelerated differentiation, all methods to date result in a mixture of RPE cells and neural retina cells, and thus require stringent selection before any therapeutic application. However, the only method of RPE selection described so far is the manual picking and expansion of pigmented cells, a time and labor consuming process. In this study, we generated a RPE specific reporter, which faithfully represents RPE cell differentiation and allows for easy cell sorting.

Lineage conversion of one somatic cell type to another is an alternative approach for generating specific cell types bypassing a pluripotent state. This approach has been successful in various cases, from neural lineages to cardiomyocytes and hepatocytes (Sancho-Martinez et al., 2012; Yi et al., 2012). Here we show that a small set of transcription factors can convert adult human fibroblasts into Best $1:$ GFP positive cells, which bear many morphological and molecular features of RPE such as the expression of Mitf, Best1, Cralbp, and Tyrp2. Moreover, these cells are able to obtain pigmentation, a typical characteristic of functional RPE cells.

Although we cannot rule out the possibility that other RPE-inducing factors have been overlooked, our study indeed established a platform to study the transcriptional network that regulates the conversion from human fibroblasts to RPE cells. Consistent with the previous reports that Mitf and Otx2 are crucial for RPE development and function (Martinez-Morales et al., 2004; Bharti et al., 2006), our data showed that Mitf and Otx2 are necessary for Best1::GFP ${ }^{+}$ colony formation. Notably, mature RPE cells are polarized, highly pigmented cells which have very limited ability for proliferation, a feature which is not suitable to be used for cell 


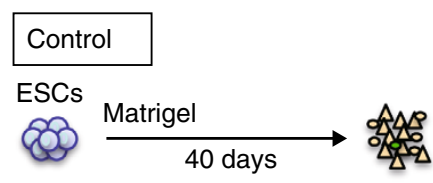

Activin A

ESCs

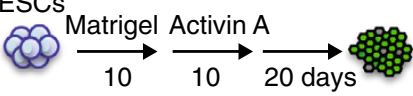

$\mathrm{RA}+\mathrm{SHH}$

ESCs

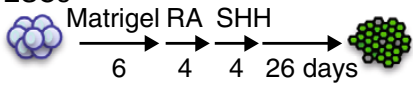

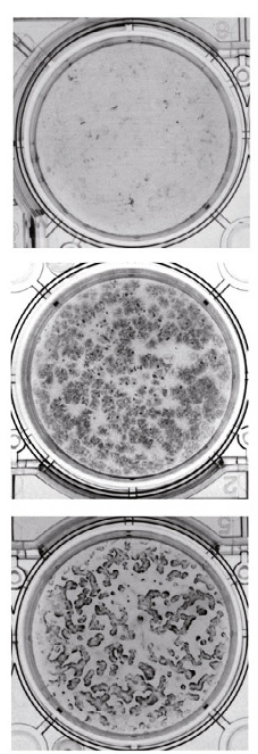

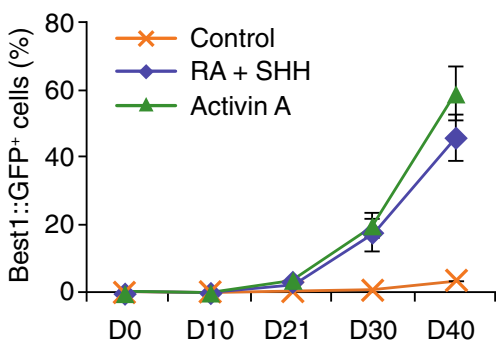

D

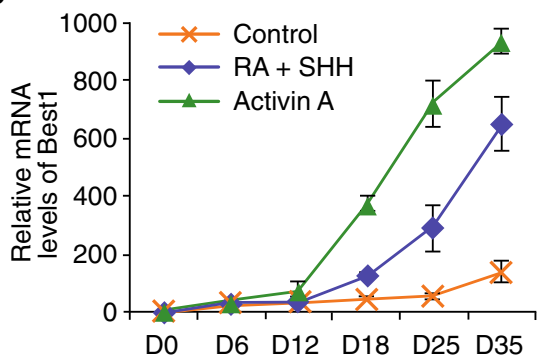

$\mathbf{E}$
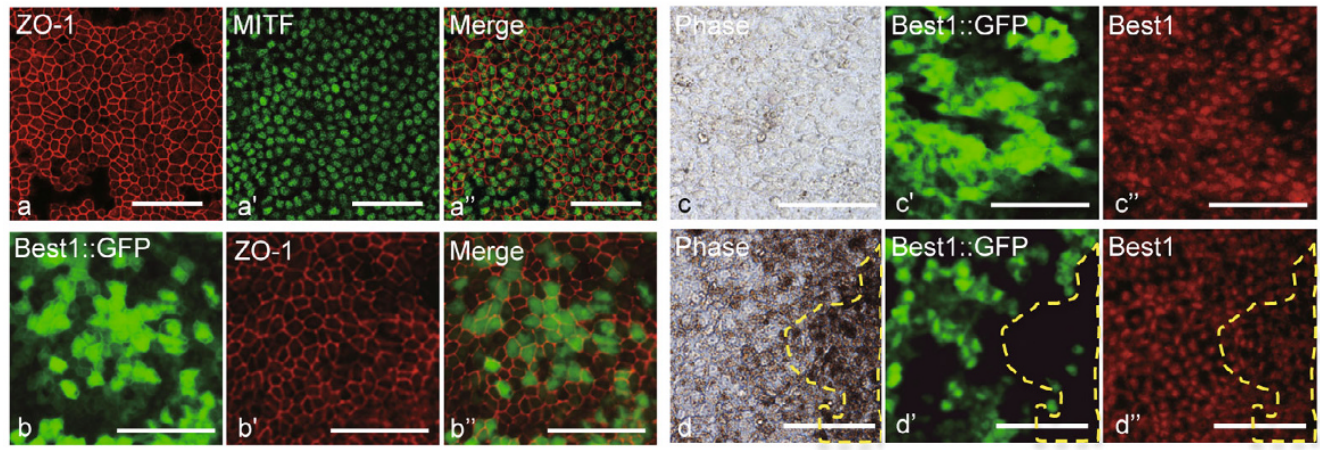

F

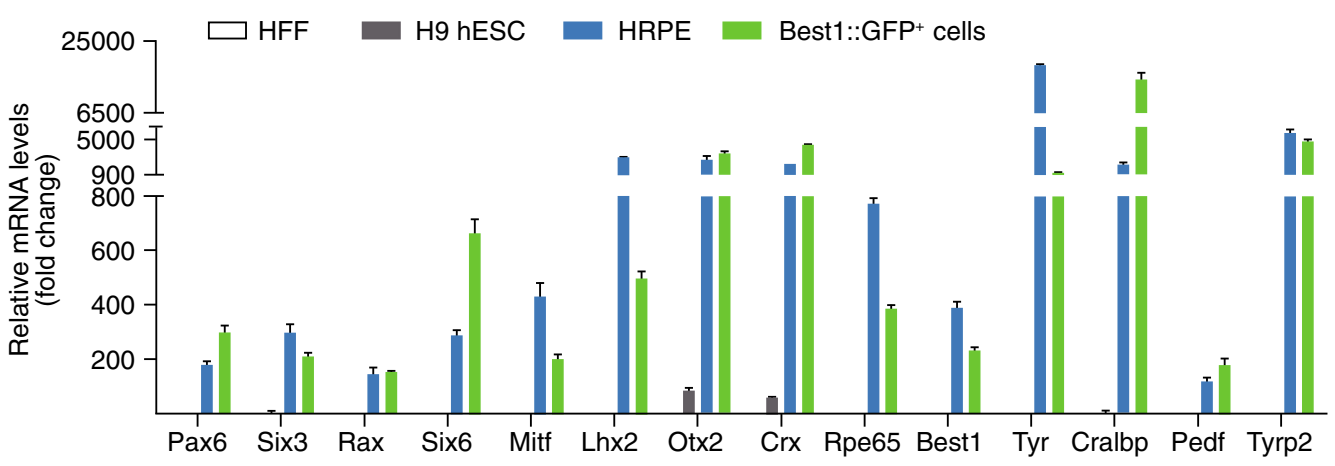

Figure 2. Characterization of Best1::GFP positive cells during hESC differentiation towards RPE. (A) Schematic illustration outlining differentiation of $\mathrm{H} 9$ hESCs with Best1::GFP reporter into RPE under different conditions. (B) Pigmentation of RPE cells differentiated from H9 hESCs using three conditions at day 40. (C) Percentages of Best1::GFP ${ }^{+}$cells calculated by FACS analysis in indicated days of H9 hESC RPE differentiation, with different treatments as indicated in Fig. 2A. Data are presented as mean \pm s.d. of three biological replicates. (D) Q-PCR analysis of Best1 mRNA expression levels in indicated days of RPE differentiation. Expression was normalized to levels in undifferentiated H9 hESC. (E) Immunofluorescence staining and phase contrast microscopy of RPE differentiation from H9 hESCs. a-a", Immunofluorescence analysis of ZO-1 and MITF expression at day 45. b-b", Best1::GFP and ZO-1 expression at day 45. c-c", Best1::GFP expression and immunostaining of Best1 protein. Best1::GFP is highly expressed in non-pigmented or slightly pigmented cells at day 30. d-d", Best1::GFP expression and immunostaining of Best1 protein at day 45. Yellow dash line highlighted area indicates diminished Best $1:$ GFP expression in heavily pigmented cells. All scale bars: $100 \mu \mathrm{m}$. (F) Relative fold changes of RPE specific genes in HFF, H9 hESCs, HRPE cells and Best1::GFP ${ }^{+}$cells which were sorted by FACS at day 40 of hESC RPE differentiation. Relative expressions were normalized to levels in HFF. 
A

\begin{tabular}{|lll|}
\hline Gene & Accession Number & Length (bp) \\
\hline Crx & NM_000554.2 & 900 \\
\hline Pax6 & NM_000280.2 & 1269 \\
\hline Mitf-A & NM_198159.2 & 1563 \\
\hline Otx2 & NM_021728.2 & 891 \\
\hline Nrl & NM_006177.2 & 714 \\
\hline Rax & NM_013435.2 & 1041 \\
\hline
\end{tabular}

B

Virus infection

(TFs pools + Best1::GFP reporter)

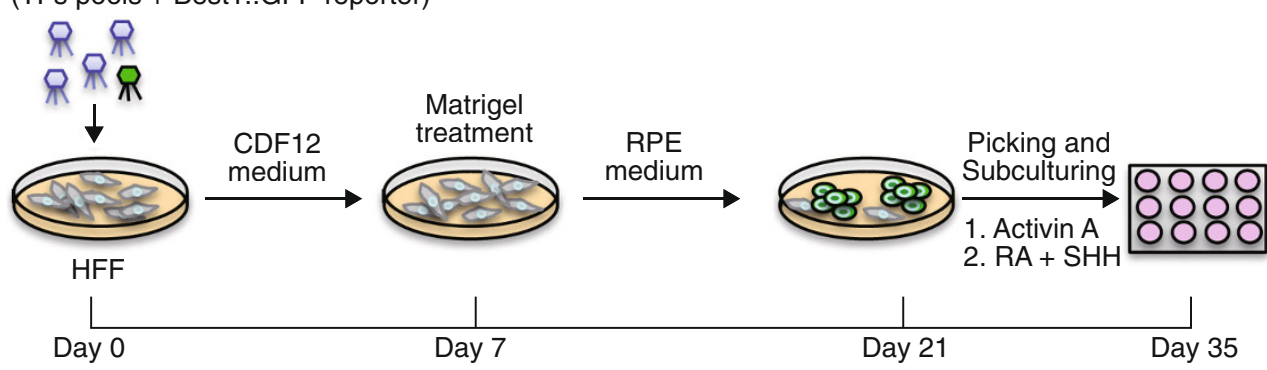

C
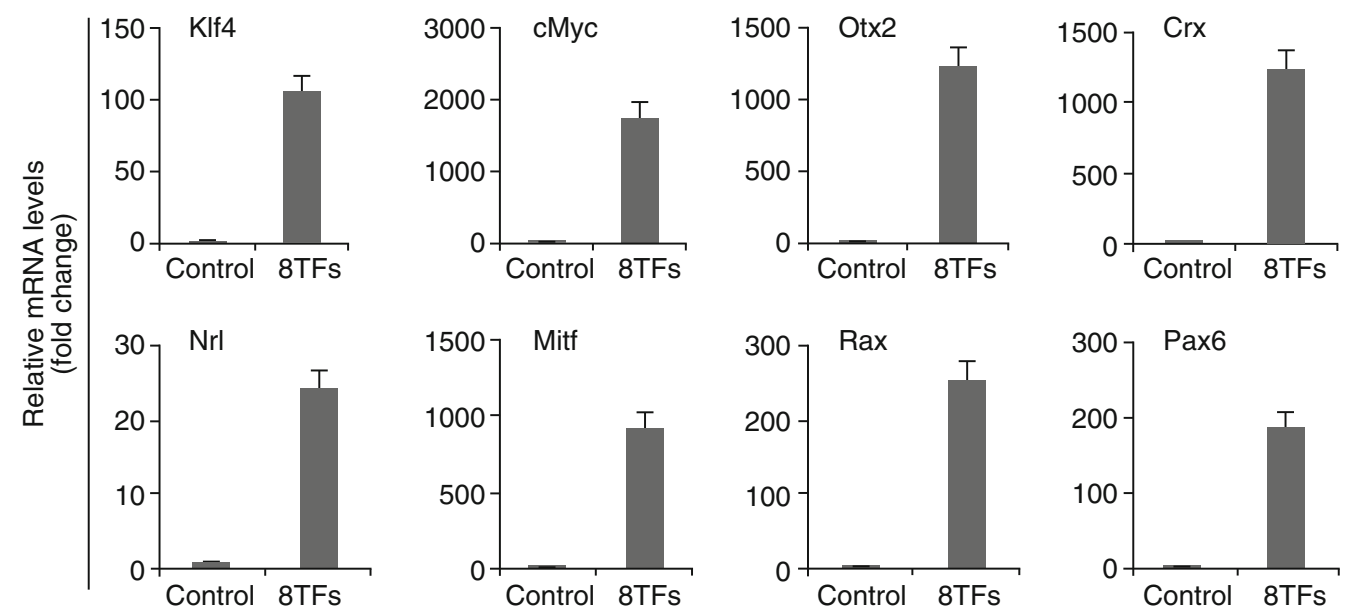

D
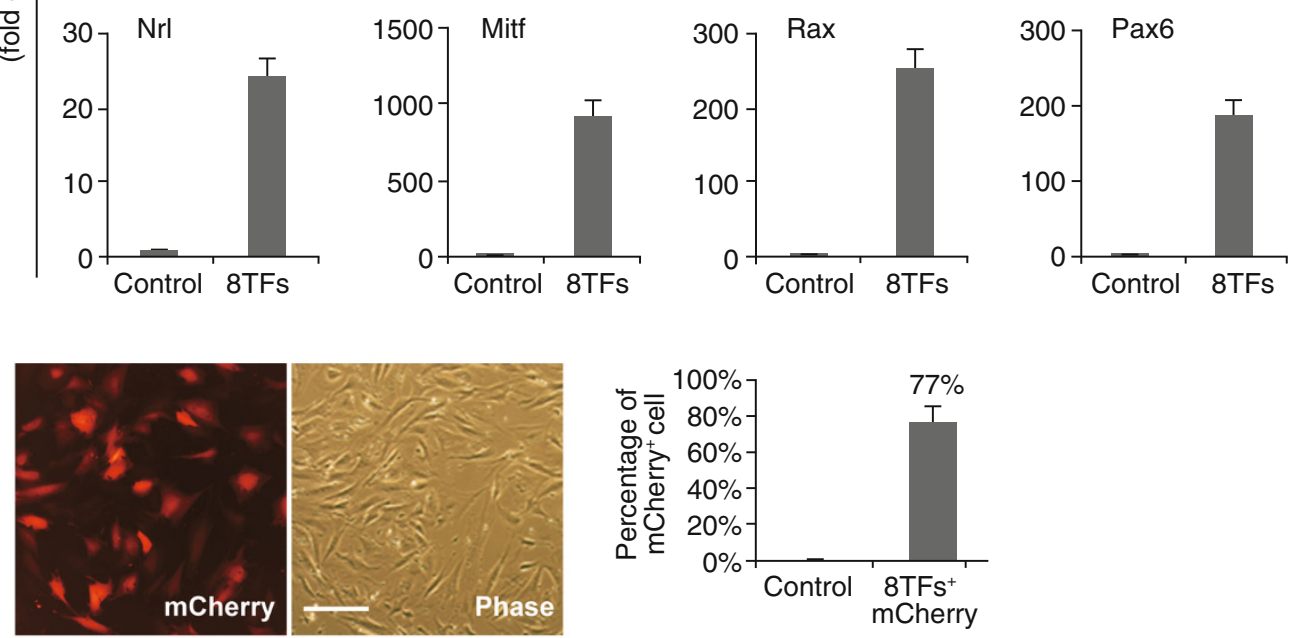

Figure 3. Strategy for transcription factors-mediated human fibroblast to RPE transdifferentiation. (A) Table listing the selected 6 transcription factors (6TFs). (B) Experimental design to induce the conversion of HFF into RPE. (C) qPCR analysis of individual exogenous TF expression 3 days after HFFs were transduced with 8TFs. HFF cells transduced with mCherry were included as the control. Data are presented as mean \pm s.d. of three replicates. (D) mCherry as indicator for the transduction efficiency of HFF cells 3 days after retroviral 8TFs plus mCherry were infected. The percentage of mCherry positive cells was indicated. Scale bars: $100 \mu \mathrm{m}$. 
A

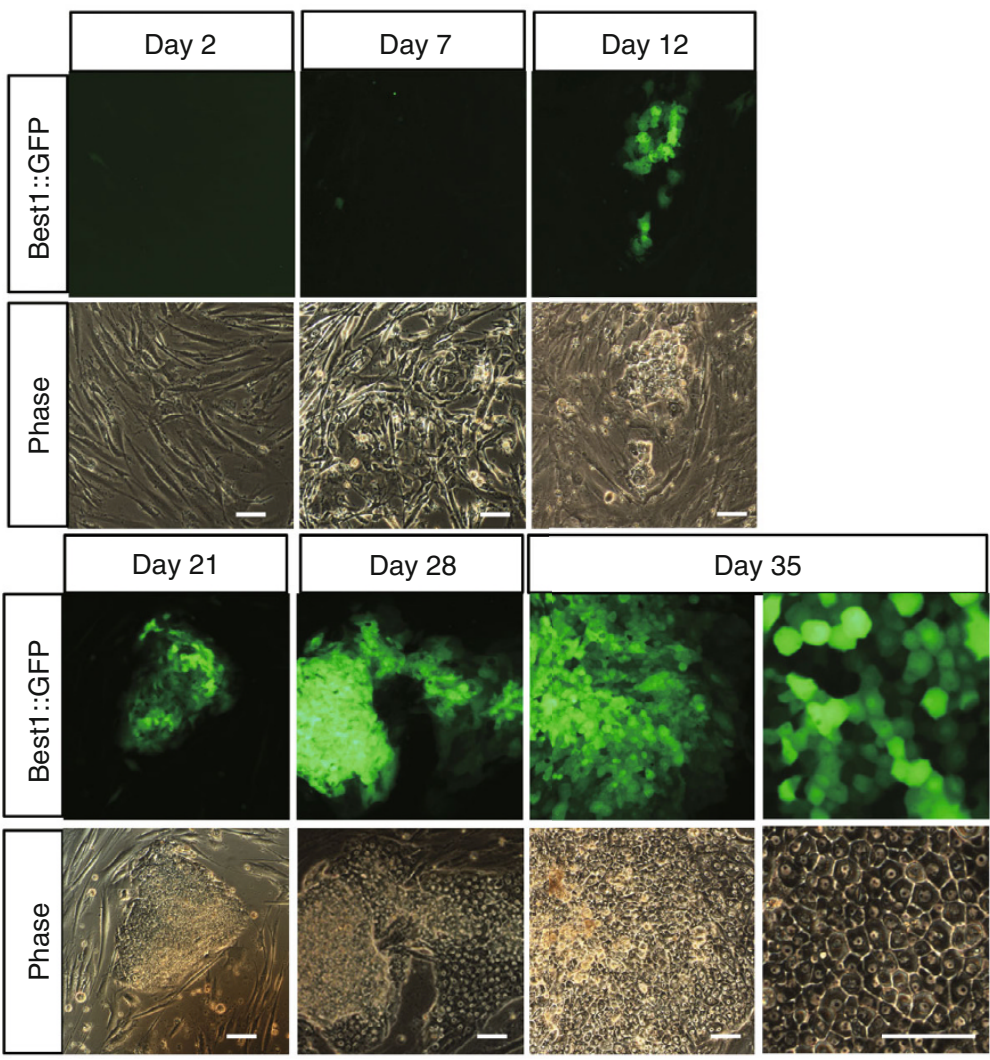

B

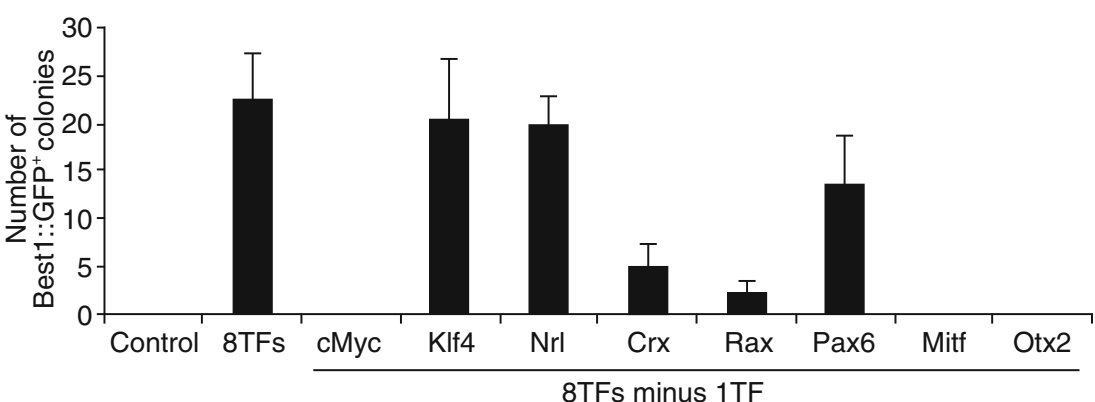

Figure 4. Eight transcription factors induced Best1::GFP ${ }^{+}$colonies from human fibroblasts. (A) Morphological changes of HFF during the process of RPE lineage conversion. Best1::GFP ${ }^{+}$cells exhibited cobblestone-like morphology at day 35 . All scale bars: $100 \mu \mathrm{m}$. (B) Effects of individual factor withdrawal from 8 transcription factors (8TFs) on Best1::GFP ${ }^{+}$colony formation. HFF cells transduced with $\mathrm{mCherry}$ were served as the control. Data are presented as mean \pm s.d. of three replicates.

transplantation (Salero et al., 2012). On the other hand, Best $1:: \mathrm{GFP}^{+}$colonies we converted from adult fibroblasts showed a progenitor-like status which is expandable. Developing an inducible expression system capable of shutting down some progenitor genes' expression at certain time points would be crucial for controlling the switch between the proliferation and maturation status of these Best $1:: G^{+} P^{+}$cells. In addition to transcription factors, culture condition has also been highlighted as a critical factor determining the lineage conversion process (Efe et al., 2011; Kurian et al., 2013). Here in this report, we provided a matrigel based culture condition combined with $\mathrm{RA}$ and $\mathrm{SHH}$ treatment that supports the generation of pigmented cells from the Best $1::$ GFP $^{+}$cells. Although we have not tested whether the RPE cells we obtained are fully functional, e.g. by cell transplantation in animal models, with further methodological optimization, this approach would facilitate the functional RPE cells' generation from human fibroblasts. In summary, our findings provide a powerful system not only for studying the molecular nature of cell identity and plasticity, but also for developing therapeutic strategies for retinal degenerative diseases. 

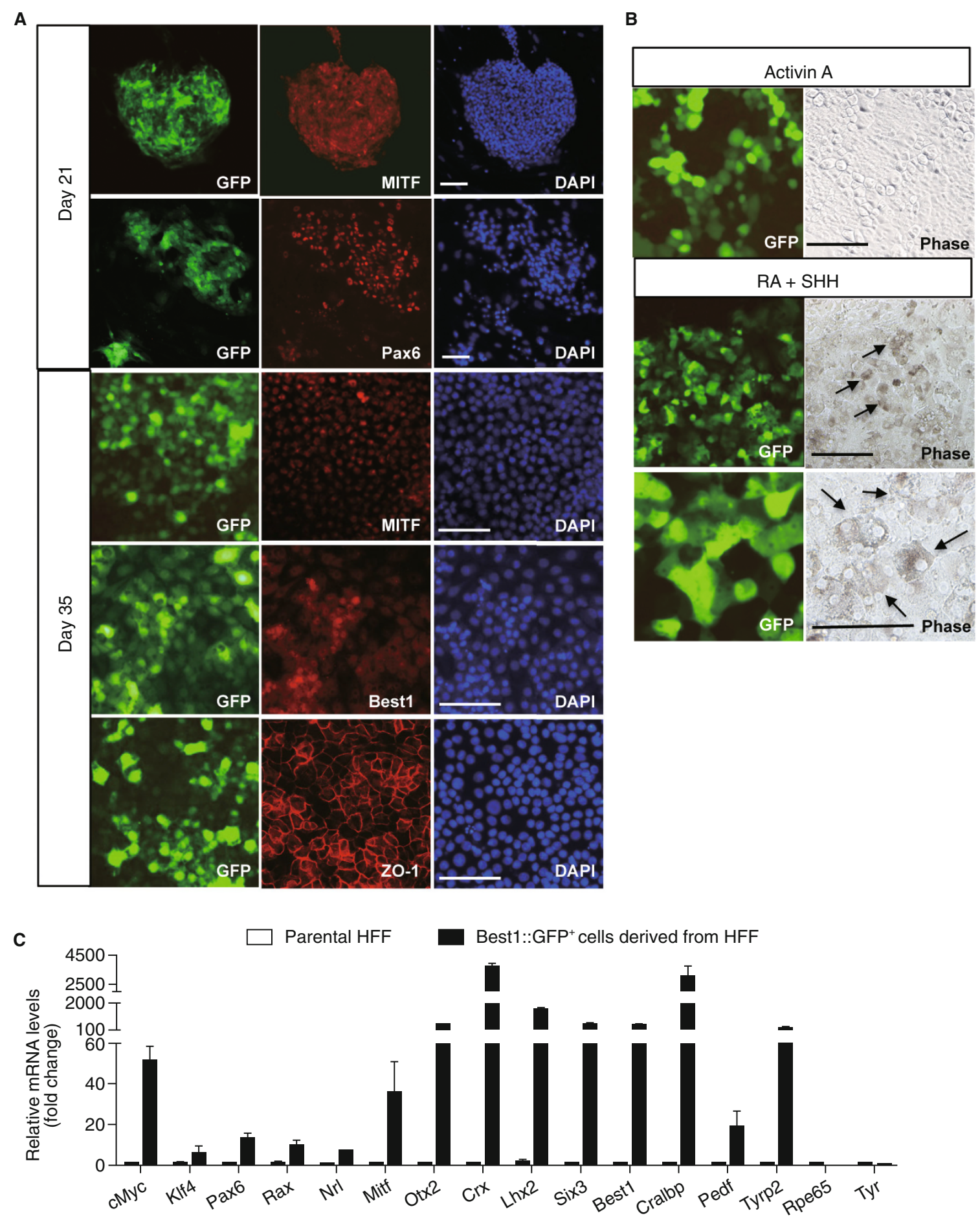

Figure 5. Characterization of Best1::GFP ${ }^{+}$cells converted from human fibroblasts. (A) Immunostaining of MITF, Pax6, Best1 and ZO-1 in Best1::GFP ${ }^{+}$colonies. DAPI was used to counterstain the nuclei (blue). All scale bars: $100 \mu \mathrm{m}$. (B) Pigmented cells indicated by arrows were observed at day 35 post-transduction in the Best1::GFP ${ }^{+}$colonies generated by treatment with RA plus $\mathrm{SHH}$, but not by Activin A treatment. All scale bars: $100 \mu \mathrm{m}$. (C) qPCR analysis of RPE-specific gene expression. 8TF-induced Best1::GFP ${ }^{+}$colonies from HFF were picked and subcultured in RA plus SHH condition. Best1::GFP ${ }^{+}$cells were sorted for qPCR analysis at day 35 . Relative expressions were normalized to levels in parental HFF. Data are presented as mean \pm s.d. of three replicates. 


\section{MATERIAL AND METHODS}

Cell culture

$\mathrm{H} 9$ and $\mathrm{H} 1 \mathrm{hESC}$ (WiCell Research) were maintained on a layer of mitotically inactivated mouse embryonic fibroblasts (MEFs) in hESC medium (CDF12 medium): DMEM/F12 (Invitrogen) supplemented with $0.1 \mathrm{mmol} / \mathrm{L}$ non-essential amino acids (Invitrogen), $1 \mathrm{mmol} / \mathrm{L}$ GlutaMAX (Invitrogen), 20\% Knockout Serum Replacement (Invitrogen), $55 \mu \mathrm{mol} / \mathrm{L} \beta$-mercaptoethanol (Invitrogen) and $10 \mathrm{ng} / \mathrm{mL}$ bFGF (Joint Protein Central). hESCs were also cultured in mTeSR medium (StemCell Technologies). Plates were coated with Matrigel (BD Biosciences), and medium was changed daily.

Human primary RPE cells (HRPE) (Lonza) used as a positive control were cultured in basic RPE medium consisting of DMEM/F12 supplemented with $0.1 \mathrm{mmol} / \mathrm{L}$ non-essential amino acids, 1 mmol/L GlutaMAX, 1\% N2 supplement (Invitrogen) and 5\% Knockout Serum Replacement (Invitrogen).

\section{RPE differentiation from hESCs}

hESCs were dissociated as cell clumps and plated on 1\% ESC qualified-Matrigel for $1 \mathrm{~h}$. Attached aggregates of cells were covered with $2 \%$ ESC qualified-Matrigel diluted in N2B27 medium consisting of DMEM/F-12 supplemented with $0.1 \mathrm{mmol} / \mathrm{L}$ non-essential amino acids, $1 \mathrm{mmol} / \mathrm{L}$ GlutaMAX, 1\% N2 supplement (Invitrogen) and 2\% B27 (Invitrogen). After overnight incubation, fresh medium without Matrigel was added and changed every other day.

For RA plus SHH treatment, Retinoic acid (RA) $(500 \mathrm{nmol} / \mathrm{L}$, Sigma) was supplemented into N2B27 medium from day 7 , then gradually reduced to $200 \mathrm{nmol} / \mathrm{L}$ by day 10 . From day 11 to day 14 , medium was changed into N2B27 medium supplemented with Sonic Hedgehog $(\mathrm{SHH})(25 \mathrm{nmol} / \mathrm{L}$, Prospec). Later, medium was changed into basic RPE medium consisting of DMEM/F-12 supplemented with $0.1 \mathrm{mmol} / \mathrm{L}$ non-essential amino acids, $1 \mathrm{mmol} / \mathrm{L}$ GlutaMAX, 1\% N2 supplement and 5\% Knockout Serum Replacement.

For Activin A treatment, the medium was changed to DMEM supplemented with $0.1 \mathrm{mmol} / \mathrm{L}$ non-essential amino acids, $1 \mathrm{mmol} / \mathrm{L}$ GlutaMAX, 20\% Knockout Serum Replacement (Invitrogen), and Activin A (100 nmol/L, Prospec) from day 10 to day 20. Later medium was changed into the basic RPE medium as described above.

\section{Production of lentivirus and retrovirus}

For generating lentiviral reporters, DNA fragments as described in Fig. 1A were obtained by PCR amplification from $\mathrm{H} 1 \mathrm{hESC}$-derived genomic DNA template and cloned into $p$ GreenZeo lentiviral vector (System bioscience). Corresponding packaging plasmids are pMDL, pCMV_VSVG and pRSV_REV. For transcription factors mediated lineage conversion, the human cDNAs listed in Fig. 3A were cloned into the pMX-gateway vector (Clontech). Coding region for mCherry was cloned as control. Corresponding packaging plasmids are pCMV-GAG-Pol and pCMV-VSV-G. HEK293T cells were seeded at a density between $6.0-8.5 \times 10^{4}$ cells $/ \mathrm{cm}^{2}$ and transfected by Lipofectamine 2000 (Invitrogen) $16 \mathrm{~h}$ later. Individual supernatants containing virus were harvested at $48 \mathrm{~h}$ and $72 \mathrm{~h}$ post-transfection and filtered with $0.45 \mu \mathrm{m}$ PVDF membrane (Millipore).
Conversion of human fibroblasts into Best1::GFP ${ }^{+}$cells

Human foreskin fibroblasts (HFF-1, HFF-693) were plated on Matrigel-coated six-well plates at 75,000 cells per well. The next day, cells were infected with an equal ratio of a combination of eight retroviruses encoding PAX6, RAX, CRX, MITF-A, OTX2, NRL, KLF4 and c-MYC (8F) as well as pGZ-BEST1-GFP lentivirus. The plates were infected by spinfection of the cells at $1850 \mathrm{r} / \mathrm{min}$ for $1 \mathrm{~h}$ at room temperature in the presence of polybrene $(4 \mu \mathrm{g} / \mathrm{mL})$ and put back in the incubator without medium change. $24 \mathrm{~h}$ later, the medium was switched to CDF12 medium with medium changes every day. Cells were covered with $2 \%$ ESC qualified-Matrigel diluted in N2B27 medium at day 7. After overnight incubation, fresh RPE medium without Matrigel was added and changed every other day. At day 21, Best $1:: \mathrm{GFP}^{+}$colonies were picked and cultured in Matrigel coated 12 well plates, followed by 10 days treatment with $100 \mathrm{ng} / \mathrm{mL}$ Activin A or $500 \mathrm{nmol} / \mathrm{L}$ RA plus $25 \mathrm{ng} / \mathrm{mL} \mathrm{SHH}$ in base RPE medium.

Immunofluorescence staining

Cells were fixed using $4 \%$ paraformaldehyde in PBS at $4^{\circ} \mathrm{C}$ for $1 \mathrm{~h}$. After fixation, cells were exposed to $0.3 \%$ Triton X-100 in PBS for 5 min at RT. Cells were blocked with $5 \%$ BSA in PBS for 30 min and incubated with primary antibody for $1 \mathrm{~h}$ at RT or overnight at $4^{\circ} \mathrm{C}$. Washing was conducted with PBS followed by incubation with a corresponding secondary antibody for $1 \mathrm{~h}$ at RT. DAPI, was used to stain nuclei. Primary antibodies were obtained from the following sources: Mouse anti-Pax6 (1:1000, DSHB); Mouse anti-MITF (1:50, Millipore); Rabbit anti-ZO-1 (1:200, Sigma); Mouse anti-Best1 (1:200, Millipore).

\section{Quantitative PCR (qPCR)}

Total cellular RNA was isolated using Trizol reagent (Invitrogen), or RNeasy Micro Kit (Qiagen) for a small amount of cell samples according to the manufacturer's recommendations. Total RNA was treated with $2 \mu \mathrm{g}$ of DNase 1 (Invitrogen) and used for cDNA synthesis using iScript reverse transcription supermix (Bio-Rad). Realtime PCR was performed using the SYBR Green PCR Master Mix (Applied Biosystems). The expression levels of respective genes were normalized to corresponding GAPDH values and are shown as fold change relative to the value of the control sample. All the assays were performed in triplicate. Primer sequences are listed in Table S1.

\section{Flow cytometry analysis and cell sorting}

H9 hESCs were infected with lentiviral Best1::GFP reporter and then underwent RPE differentiation. Cells were harvested using TrypLE (Invitrogen), washed once with PBS and resuspended with $1 \times$ PBS/ $10 \%$ FCS medium. A minimum of 10,000 cells in the living population were analyzed by using a BD LSRII flow cytometry machine equipped with five different lasers and the BD FACSDiva software. Percentages of Best $1:: \mathrm{GFP}^{+}$cells are presented after the subtraction of isotype background and refer to the total living population analyzed. Results are representative of at least three independent experiments with a minimum of two technical replicates per 
experiment. For cell sorting, cells were treated as described above and sorted with a BDAria II FACS sorter (BD Biosystems).

\section{ACKNOWLEDGEMENTS}

G.H.L. is supported by the Strategic Priority Research Program of the Chinese Academy of Sciences (XDA01020312), National Natural Science Foundation of China (Grant Nos. 81271266, 31222039, and 31201111), the Thousand Young Talents program of China, National Laboratory of Biomacromolecules (2013kf05, and 2013kf11), and State Key Laboratory of Drug Research (SIMM1302KF-17). JCIB was supported by CIBER-BBN and grants from MINECO, Fundacion Cellex, G. Harold and Leila Y. Mathers Charitable Foundation, The Leona M. and Harry B. Helmsley Charitable Trust and The Ellison Medical Foundation.

\section{ABBREVIATIONS}

AMD, age-related macular degeneration; ESC, embryonic stem cell; FACS, fluorescence activated cell sorting; iPSC, induced pluripotent stem cell; MEF, mouse embryonic fibroblast; RA, retinoic acid; RPE, retinal pigment epithelium; $\mathrm{SHH}$, Sonic Hedgehog; TF, transcription factor.

\section{COMPLIANCE WITH ETHICS GUIDELINES}

All procedures followed were in accordance with the ethical standards of the responsible committee on human experimentation (Center of Regenerative Medicine in Barcelona, Spain) and with the Helsinki Declaration of 1975, as revised in 2000 (5). Informed consent was obtained from all patients for being included in the study.

Kejing Zhang, Guang-Hui Liu, Fei Yi, Nuria Montserrat, Tomoaki Hishida, Concepcion Rodriguez Esteban, Juan Carlos Izpisua Belmonte declare that they have no conflict of interest.

\section{OPEN ACCESS}

This article is distributed under the terms of the Creative Commons Attribution License which permits any use, distribution, and reproduction in any medium, provided the original author(s) and the source are credited.

\section{REFERENCES}

Acland GM, Aguirre GD, Bennett J, Aleman TS, Cideciyan AV, Bennicelli J, Dejneka NS, Pearce-Kelling SE, Maguire AM, Palczewski K et al (2005) Long-term restoration of rod and cone vision by single dose rAAV-mediated gene transfer to the retina in a canine model of childhood blindness. Mol Ther 12:1072-1082

Ben-David U, Benvenisty N (2011) The tumorigenicity of human embryonic and induced pluripotent stem cells. Nat Rev Cancer 11:268-277

Bharti K, Nguyen MT, Skuntz S, Bertuzzi S, Arnheiter H (2006) The other pigment cell: specification and development of the pigmented epithelium of the vertebrate eye. Pigment Cell Res 19:380-394

Boucherie C, Mukherjee S, Henckaerts E, Thrasher AJ, Sowden JC, Ali RR (2013) Brief report: self-organizing neuroepithelium from human pluripotent stem cells facilitates derivation of photoreceptors. Stem Cells 31:408-414
Boulanger A, Liu S, Henningsgaard AA, Yu S, Redmond TM (2000) The upstream region of the Rpe65 gene confers retinal pigment epithelium-specific expression in vivo and in vitro and contains critical octamer and E-box binding sites. J Biol Chem 275:3127431282

Buchholz DE, Hikita ST, Rowland TJ, Friedrich AM, Hinman CR, Johnson LV, Clegg DO (2009) Derivation of functional retinal pigmented epithelium from induced pluripotent stem cells. Stem Cells 27:2427-2434

Carr AJ, Vugler AA, Hikita ST, Lawrence JM, Gias C, Chen LL, Buchholz DE, Ahmado A, Semo M, Smart MJ et al (2009) Protective effects of human iPS-derived retinal pigment epithelium cell transplantation in the retinal dystrophic rat. PLoS ONE 4: e8152

Efe JA, Hilcove S, Kim J, Zhou H, Ouyang K, Wang G, Chen J, Ding $S$ (2011) Conversion of mouse fibroblasts into cardiomyocytes using a direct reprogramming strategy. Nat Cell Biol 13:215-222

Esumi N, Oshima Y, Li Y, Campochiaro PA, Zack DJ (2004) Analysis of the VMD2 promoter and implication of E-box binding factors in its regulation. J Biol Chem 279:19064-19073

Esumi N, Kachi S, Hackler L Jr, Masuda T, Yang Z, Campochiaro PA, Zack DJ (2009) BEST1 expression in the retinal pigment epithelium is modulated by OTX family members. Hum Mol Genet 18:128-141

Giorgetti A, Marchetto MC, Li M, Yu D, Fazzina R, Mu Y, Adamo A, Paramonov I, Cardoso JC, Monasterio MB et al (2012) Cord blood-derived neuronal cells by ectopic expression of Sox2 and c-Myc. Proc Natl Acad Sci USA 109:12556-12561

Huang P, He Z, Ji S, Sun H, Xiang D, Liu C, Hu Y, Wang X, Hui L (2011) Induction of functional hepatocyte-like cells from mouse fibroblasts by defined factors. Nature 475:386-389

Khandhadia S, Cherry J, Lotery AJ (2012) Age-related macular degeneration. Adv Exp Med Biol 724:15-36

Kim J, Efe JA, Zhu S, Talantova M, Yuan X, Wang S, Lipton SA, Zhang K, Ding $S$ (2011) Direct reprogramming of mouse fibroblasts to neural progenitors. Proc Natl Acad Sci USA 108:7838-7843

Kurian L, Sancho-Martinez I, Nivet E, Aguirre A, Moon K, Pendaries C, Volle-Challier C, Bono F, Herbert JM, Pulecio J et al (2013) Conversion of human fibroblasts to angioblast-like progenitor cells. Nat Methods 10:77-83

Liu GH, Yi F, Suzuki K, Qu J, Izpisua Belmonte JC (2012) Induced neural stem cells: a new tool for studying neural development and neurological disorders. Cell Res 22:1087-1091

Lu B, Malcuit C, Wang S, Girman S, Francis P, Lemieux L, Lanza R, Lund R (2009) Long-term safety and function of RPE from human embryonic stem cells in preclinical models of macular degeneration. Stem Cells 27:2126-2135

Martinez-Morales JR, Rodrigo I, Bovolenta P (2004) Eye development: a view from the retina pigmented epithelium. BioEssays 26:766-777

Meyer JS, Shearer RL, Capowski EE, Wright LS, Wallace KA, McMillan EL, Zhang SC, Gamm DM (2009) Modeling early retinal development with human embryonic and induced pluripotent stem cells. Proc Natl Acad Sci USA 106:16698-16703

Nakano T, Ando S, Takata N, Kawada M, Muguruma K, Sekiguchi K, Saito K, Yonemura S, Eiraku M, Sasai Y (2012) Self-formation of 
optic cups and storable stratified neural retina from human ESCs. Cell Stem Cell 10:771-785

Okada Y, Shimazaki T, Sobue G, Okano H (2004) Retinoic-acidconcentration-dependent acquisition of neural cell identity during in vitro differentiation of mouse embryonic stem cells. Dev Biol 275:124-142

Osakada F, Ikeda H, Sasai Y, Takahashi M (2009) Stepwise differentiation of pluripotent stem cells into retinal cells. Nat Protoc 4:811-824

Pang ZP, Yang N, Vierbuchen T, Ostermeier A, Fuentes DR, Yang TQ, Citri A, Sebastiano V, Marro S, Sudhof TC et al (2011) Induction of human neuronal cells by defined transcription factors. Nature 476:220-223

Panopoulos AD, Ruiz S, Izpisua Belmonte JC (2011) iPSCs: induced back to controversy. Cell Stem Cell 8:347-348

Salero E, Blenkinsop TA, Corneo B, Harris A, Rabin D, Stern JH, Temple S (2012) Adult human RPE can be activated into a multipotent stem cell that produces mesenchymal derivatives. Cell Stem Cell 10:88-95

Sancho-Martinez I, Baek SH, Izpisua Belmonte JC (2012) Lineage conversion methodologies meet the reprogramming toolbox. Nat Cell Biol 14:892-899

Schwartz SD, Hubschman JP, Heilwell G, Franco-Cardenas V, Pan CK, Ostrick RM, Mickunas E, Gay R, Klimanskaya I, Lanza R (2012) Embryonic stem cell trials for macular degeneration: a preliminary report. Lancet 379:713-720

Sekiya S, Suzuki A (2011) Direct conversion of mouse fibroblasts to hepatocyte-like cells by defined factors. Nature 475:390-393

Steingrimsson E, Copeland NG, Jenkins NA (2004) Melanocytes and the microphthalmia transcription factor network. Annu Rev Genet 38:365-411
Strauss $O$ (2005) The retinal pigment epithelium in visual function. Physiol Rev 85:845-881

Takahashi K, Yamanaka S (2006) Induction of pluripotent stem cells from mouse embryonic and adult fibroblast cultures by defined factors. Cell 126:663-676

Takahashi K, Tanabe K, Ohnuki M, Narita M, Ichisaka T, Tomoda K, Yamanaka S (2007) Induction of pluripotent stem cells from adult human fibroblasts by defined factors. Cell 131:861-872

Vierbuchen T, Ostermeier A, Pang ZP, Kokubu Y, Sudhof TC, Wernig $M$ (2010) Direct conversion of fibroblasts to functional neurons by defined factors. Nature 463:1035-1041

Yi F, Liu GH, Izpisua Belmonte JC (2012) Rejuvenating liver and pancreas through cell transdifferentiation. Cell Res 22:616-619

Zahabi A, Shahbazi E, Ahmadieh H, Hassani SN, Totonchi M, Taei A, Masoudi N, Ebrahimi M, Aghdami N, Seifinejad A et al (2012) A new efficient protocol for directed differentiation of retinal pigmented epithelial cells from normal and retinal disease induced pluripotent stem cells. Stem Cells Dev 21:2262-2272

Zhang W, Duan S, Li Y, Xu X, Qu J, Zhang W, Liu GH (2012) Converted neural cells: induced to a cure? Protein Cell 3:91-97

Zhang K, Yi F, Liu GH, Izpisua Belmonte JC (2013) New march towards the regeneration of sensation and cognition: hear more, see more and learn more. J Mol Cell Biol 5:151-153

Zhu Y, Carido M, Meinhardt A, Kurth T, Karl MO, Ader M, Tanaka EM (2013) Three-dimensional neuroepithelial culture from human embryonic stem cells and its use for quantitative conversion to retinal pigment epithelium. PLoS ONE 8:e54552

Zuber ME, Gestri G, Viczian AS, Barsacchi G, Harris WA (2003) Specification of the vertebrate eye by a network of eye field transcription factors. Development 130:5155-5167 